\title{
The Diffusion of Vegan Food Innovations: A Dual-market Perspective
}

\author{
*Ozgur Dedehayir \\ School of Management, Queensland University of Technology, Brisbane, Australia \\ e-mail: ozgur.dedehayir@qut.edu.au \\ Carla Riverola \\ La Salle - Universitat Ramon Llull, Barcelona, Spain \\ e-mail: criverola@salleurl.edu \\ Santiago Velasquez \\ School of Management, Universidad de Los Andes, Bogotá, Colombia \\ e-mail: sa.velasquezf@uniandes.edu.co \\ Michelle Smidt \\ School of Management, Queensland University of Technology, Brisbane, Australia \\ e-mail: m.smidt@qut.edu.au \\ *corresponding author
}

\section{Definitions}

Veganism is the practice of abstaining from the use of animal products, whether it is for food, clothing, or otherwise. Individuals adhering to veganism are referred to as vegans, and the products they choose to purchase or consume are absent of ingredients sourced from animals.

Innovations are products that are appreciated as new and novel by consumers. Vegan food innovations subsequently refer to vegan food products that are seen as new and novel among potential consumers.

After their initial introduction to the market, innovations are purchased, or adopted, by a growing number of consumers over time. The process through which the innovation reaches the hands of more and more adopters is referred to as diffusion.

Keywords: innovation; new product development; vegan; diffusion; sustainable; food innovation

\section{Introduction}

The intense agricultural activity required to satisfy the greater demand of an increasing global population relies on non-renewable resources, such as fossil fuels and land. Given that $33 \%$ of the planet's arable land is used to grow crops for animal feed (Adams 2010), and approximately $70 \%$ of agricultural land on this planet is devoted to livestock production (Deckers 2009, Judge and Wilson 2015), the meat and dairy industry accounts for a significant part of this problem. While notable attention has been afforded to technological solutions for increasing the efficiency of land use and reduction of harmful emissions in the meat and dairy industry - hence, 'supply-side' solutions - this paper considers solutions that can arise from the 'demand-side', in other words, from the consumers themselves. To this end, the paper turns its attention to the ethical eating advocated by 'veganism', which strives to alleviate the suffering of animals, particularly those subjected to ill-treatment in the meat and dairy industry (Singer 1990), by abstaining from the consumption of goods that have used animals at any stage of their production. The paper argues that veganism not only protects animal rights, but may also offer a solution to some of the systemic problems of the global food system. 
Although individuals following a vegan lifestyle form a minority in the developed world, recent trends indicate notable growth in their numbers. For instance, the number of vegans in the USA has increased from 300,000-500,000 in 1997, to 2.5-6 million in 2012 (1-2\% of the population). Similar ratios and trends have been reported in other countries, such as Israel (5\%), the UK (2\%), Australia $(1 \%)$, and Germany $(1 \%)$. Given the ethical motivations underlying veganism, the products and services (e.g. food, clothing, and cosmetics) adopted by vegans must be absent of animal sourced ingredients (see Singer 1990, for a comprehensive review). This requirement has inevitably created niches within different markets (e.g. Greenebaum 2012), with the noted growth in these niches attracting new business ventures to serve the greater demand through new vegan products and services (i.e. vegan innovations).

Against this backdrop, the paper aims to understand the factors that can drive the dissemination of vegan innovations, specifically, new products in vegan food manufacturing and retail, beyond these niche segments into the mainstream market. To acquire an understanding of these factors, the investigation is anchored in the 'diffusion of innovations' (DOI) theory developed by Everett Rogers in his book with the same title first published in 1962. Rather than a relatively smooth 'bell-curved' pattern theorized by Rogers, however, it is anticipated that the diffusion pattern of vegan food innovations will demonstrate a noticeable disparity between existing niche segments that predominantly serve vegan consumers, and the much larger segments that comprise non-vegans. This expectation of a 'dual-market' pattern resulting from the 'chasm' (or 'saddle') separating the early and main markets (Moore 1991, Kohli et al. 1999, Muller and Yogev 2006) is premised on (i) constrained design principles of vegan food innovations that limit their alignment with the consumption paradigm of traditional (i.e. non-vegan) segments; (ii) the negative sentiments of the mainstream media's coverage of veganism that may discourage adoption considerations of the main market (Cole and Morgan 2011, Taylor 2013); and (iii) the innate rejection of vegan food innovations by a significant portion of potential adopters, namely, males (Adams 2010; Potts and Parry 2010; Ruby 2012). Under these circumstances, the paper's ambition is to shed light on how vegan food innovations can propagate within the entire social system that includes both vegan and non-vegans, and the strategies available for businesses to navigate the disparity between the early and main markets.

\section{The Dual-Market Model}

The literature studying chasms, saddles, and dual-markets examines various product categories, in both the business-to-business (B2B) setting as well as the final consumer markets. A notable percentage of cases examined by scholars appear to be high-tech innovations, which diffuse within the B2B context. These studies subsequently align with the nature of a majority of cases analysed by Moore (1991) himself. They include information and communication technologies (ICTs) (Easingwood et al. 2006, Gombault et al. 2016), telehealth/medicine innovations (Cho et al. 2008, LeRouge and Garfield 2013), medical equipment (Hienerth and Lettl 2011), environmentally responsible manufacturing (Sroufe et al. 2000), energy conservation innovations for housing (Egmond et al. 2006), and CO2-saving power train technologies (Seitz et al. 2015). While scholarly contributions have also examined the chasm in business-to-consumer (B2C) contexts, many cases still fall into the high-tech product category. These include consumer electronics such as personal computers, video cassette recorders, cellular phones, laser printers, and wearable devices (e.g. Taylor et al. 1994, Goldenberg et al. 2002, Golder and Tellis 2004, Jee and Sohn 2015, Kim et al. 2016), domestic solar power systems (Faiers and Neame 2006), pharmaceutical drugs (Vakratas and Kolsarici 2008, Guseo and Guidolin 2011), satellite Digital Multimedia Broadcasting (Sawng et al. 2009), sporting equipment (Hienerth and Lettl 2011), cohousing (Williams 2008), energy-efficient homes (Sanderford et al. 2015), and fashion innovations (Zhang and Di Benedetto 2010).

Much of this prior research centres on the reasons why chasms and saddles emerge. Nevertheless, the focus of empirical studies on high-tech innovations limits our understanding of underlying causes to those that are predominantly technology-centric, such as product functionality, technological novelty, 
and price (e.g. Chandrasekaran and Tellis 2011). The scope of strategies available for businesses to navigate the disparity between the early and main markets are subsequently limited to those addressing these causes. The conceptual understanding of chasms, saddles, and dual-markets can therefore be enhanced by examining a wider scope of products. For instance, the chasm described by Moore (1991) for high-tech products arises from the complete disconnect between the early and main markets, which have radically different performance requirements.

For all intents and purposes, the literature employs the notion of a chasm or saddle as a standard, unitary phenomenon. From a business perspective, however, the characteristics of the disparity between the early and main markets are important to understand for strategic manoeuvring. For instance, the size of the chasm can indicate the motivations of the main market to adopt the innovation, and subsequently the strategic approach needed by the innovating firm to transition across the gulf. When the chasm is relatively small we may anticipate the early and main markets to share commonalities in their product requirements (Faiers and Neame 2006) and subsequently a closer word-of-mouth connection between these markets (hence, encroaching upon Rogers's depiction of a smooth diffusion process). Consequently, the strategic orientation of the innovating firm towards the early as well as the main market may overlap substantially. By contrast, when there is a relatively large disparity between the early and main markets (hence, depicting Moore's perturbed diffusion process), the strategic orientation of the firm towards each of these markets may need to be radically different. Although a few scholarly works have considered the varying magnitude of chasms and saddles (e.g. Goldenberg et al. 2002, Faiers and Neame 2006), just how this magnitude influences the diffusion of innovations has not received sufficient attention.

The cases examined in studies of chasms, saddles, and dual markets focus on products that are purchased infrequently (in the case of some, such as solar power systems, perhaps only once) due to their usage longevity. Even when these 'consumer durables' require replacement at future points in time (e.g. white goods and consumer electronics), their diffusion patterns are only studied for the first occasion of acquisition (e.g. Bass 1969). Studies do not consider the repeat purchases of these products, which would provide a more accurate understanding of adoption (Rogers 1995). And yet, many products have short useful lives after purchase and require repeated purchase decision making (e.g. fast-moving consumer goods such as cosmetics, food, medication, and clothing). Understanding the diffusion of these nondurable innovations within a social system requires an appreciation of adopters' re-evaluation of the innovation each time a purchase decision needs to be made. This is particularly important from a business perspective as the success of such innovations cannot be measured through the first time they are adopted by a group of individuals. Rather, the success (e.g. market share, revenues, and profits) derived by the firm from a nondurable innovation is bestowed by its sustained adoption in the marketplace.

\section{Methods}

To address the conceptual gaps identified from the above review of the literature - need for more empirical studies focusing on: (i) dual-markets; (ii) chasm magnitude; and (iii) diffusion of nondurable products - we have selected a particular type of food innovation, namely, 'vegan food innovations', which diffuse within a dual-market emphasised by a large divide between the early and main markets. Vegan food innovations are products created and commercialised by vendors such as restaurants and cafés (i.e. menu items), adhering to veganism principles and therefore absent of ingredients sourced from animals. Although in an historical context, creating food that is absent of animal products may not appear to be an innovation in itself, in the context of today's global and industrial food system, such purposeful consumption, or what has been termed 'mindful eating', has emerged as highly novel.

There are salient factors that help create the large disparity between the early and main markets for vegan food innovations to navigate across. First, the application of vegan philosophy to food innovations means that new products will be developed under a set of restrictions (i.e. no meat or dairy ingredients can be used). While all new products are developed with respect to some design 
principles, the vegan philosophy precludes a large number of ingredients from the product development process that are entrenched as being traditional and essential to food innovations by large segments of the market (i.e. non-vegans). Attributes of vegan food innovations are therefore likely to create an intrinsic challenge to attract the main market of adopters. Secondly, veganism has been placed under a negative spotlight in the mainstream media for a long period of time (Cole and Morgan 2011, Taylor 2013). Given that media and communication play an inherent role in diffusing innovations to larger market segments, the adoption behaviour of the main market under the influence of negative media, or negative 'hype', is likely to be curbed substantially. It is therefore anticipated that the negative media spotlight helps create a strong divide between the early and main markets. Thirdly, studies of veganism emphasise the association between meat consumption and masculinity, thus challenging the male population's transition to veganism when meat-based diets are embedded with culture mores of 'manliness' (Adams 2010, Potts and Parry 2010, Ruby 2012). Prior studies investigating the diffusion of consumable products (e.g. white goods, and brown goods such as CDs) also show cultural factors such as gender identity to be influential (Peres et al. 2010). We therefore expect males to have reservations in adopting vegan food innovations, which will further widen the disparity between the early and main markets.

To describe the dual-market resulting from this wide chasm, this study follows the lead of Taylor et al. (1994), and makes the assumption that vegans will have greater motivation to adopt vegan food innovations in comparison to non-vegans, and will therefore adopt these innovations earlier. Hence, the early market is associated with vegans and the main market with non-vegans (Figure 1).

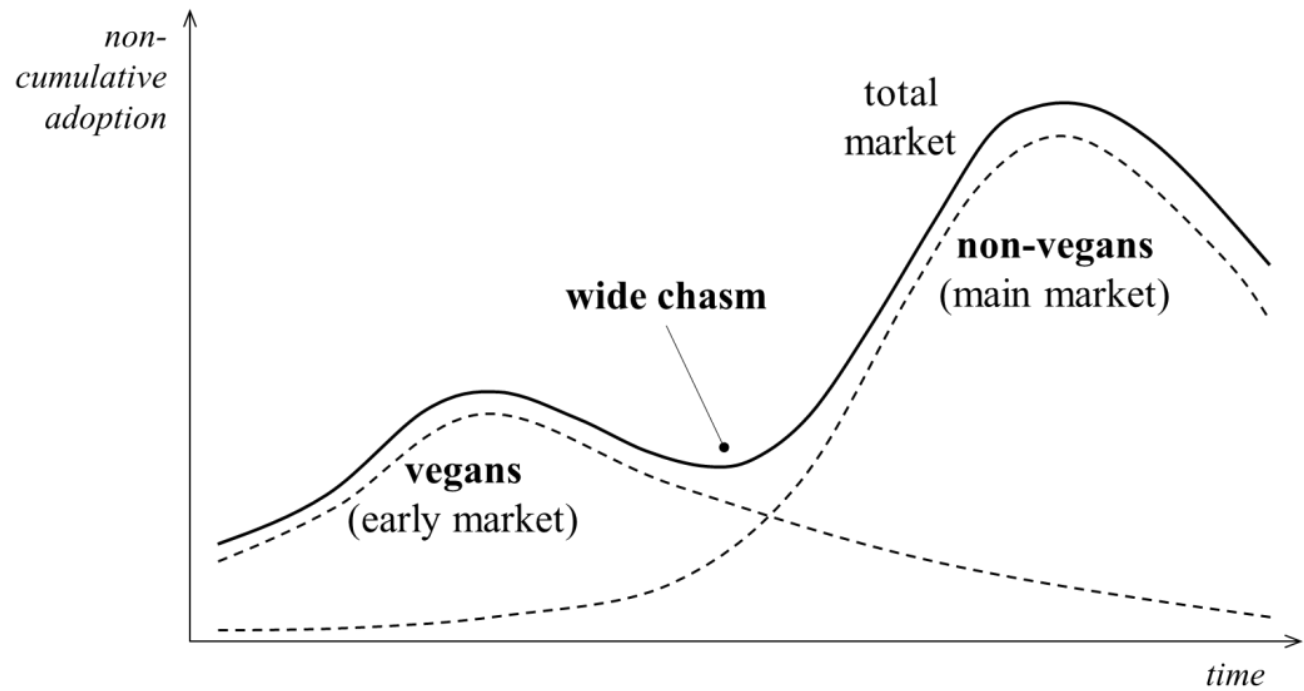

Figure 1: The dual-market of vegan food innovations and the wide chasm separating vegans (early market) and non-vegans (main market).

While these starting premises may limit analysis, the assumptions can be justified by the fact that vegan food innovations adhere to a set of design principles, which appeal directly to vegan consumers, who are inherently unable to consume non-vegan food innovations. Vakratas and Kolsarici (2008) demonstrate such a high degree of product-market alignment in their analysis of prescription drug diffusion. The authors show that a dual-market can be expected for new prescription pharmaceuticals, with the early market constituting patients with severe health problems and for whom the drug has been developed. The main market, by contrast, constitutes patients with a mild condition who have a choice of adopting the new drug or not. The findings of Vakratas and Kolsarici (2008) can be extrapolated to suggest that the design of vegan food innovations aligns directly with the needs of a certain group of individuals, namely, vegans, who are likely to be earliest adopters. In turn, non-vegans, akin to patients with mild health conditions, are not restricted to adopting vegan food innovations, and will do so only out of choice and most likely later than vegans. Similar dual- 
market structures are likely to arise in contexts governed by strong compatibility requirements between the innovation and social, cultural, and religious norms.

The paper employed a multiple case study design (Eisenhardt 1989, Miles et al. 2014), which allowed the implementation of a replication logic (Yin 1994). Despite the large number of scholarly works that test hypotheses developed by diffusion theory, the conceptual understanding of how innovations diffuse in a dual-market setting is still underdeveloped, thus motivating the implementation of an inductive, loosely designed research agenda (Edmondson and McManus 2007, Miles et al. 2014). The cases included in this study are restaurants and cafés, drawn from a population of businesses that serve vegan food innovations. For each case, interviews were conducted with restaurant and café business owners (i.e. the providers of vegan food innovations) to acquire insights on the strategic considerations of the respondents to reach the main market with their vegan food innovations. To accommodate for contextual factors that may influence our findings, case studies were conducted in three different cultural settings - Brisbane (Australia), Barcelona (Spain), and Bogotá (Colombia) with six restaurants/cafés selected in total (two in each city) through a purposive sampling strategy (Miles et al. 2014). The selection process has been theory-driven, based on the weighting of vegan food items appearing on restaurant menus. To allow for comparability across cultural settings, consistency was ensured in the menu offerings of the restaurants/cafés, which list either vegetarian or vegan dishes.

Interviews with restaurant/café owners were approximately 45 minutes in length and semi-structured. Following Wejnert's (2002) synthesis of the diffusion of innovations, the interviews were structured to comprise four sections. The first section focused on the characteristics of the business owner and his/her environment, the second addressed the business owner's perceptions of vegan food innovations and customers, the third focused on the business itself, and the fourth component centred on the business strategy. The data acquired from these interviews were supplemented by secondary data sourced from the websites of the businesses as well as documents such as menus.

\section{Restauranteurs' Perceptions of the Market}

The overview of the case analyses presented in Table 1 shows that the tenure of the business owners in their current role range from eight months to eight years. While some of the respondents had owned a business previously, most came into the sector from very different backgrounds. The novelty of running a restaurant business was epitomized by a couple of respondents who claimed to have had to learn how to cook in order to run the restaurant.

Table 1: Overview of respondents.

\begin{tabular}{|c|c|c|c|c|c|c|}
\hline & $\begin{array}{l}\text { Restaurant } \\
\text { A } \\
\text { (Brisbane) }\end{array}$ & $\begin{array}{l}\text { Restaurant } \\
\text { B } \\
\text { (Brisbane) }\end{array}$ & $\begin{array}{l}\text { Restaurant } \\
\text { C } \\
\text { (Bogotá) }\end{array}$ & $\begin{array}{l}\text { Restaurant } \\
\text { D } \\
\text { (Bogotá) }\end{array}$ & $\begin{array}{l}\text { Restaurant } \\
\text { E } \\
\text { (Barcelona) }\end{array}$ & $\begin{array}{l}\text { Restaurant } \\
\text { F } \\
\text { (Barcelona) }\end{array}$ \\
\hline gender & Male & Female & Female & Female & Male & Male \\
\hline tenure & 1.5 years & 7 years & 0.5 years & - & 8 years & 3.5 years \\
\hline $\begin{array}{l}\text { previous } \\
\text { business }\end{array}$ & Yes & Yes & No & No & - & - \\
\hline background & - & $\begin{array}{l}\text { fashion } \\
\text { designer }\end{array}$ & psychologist & nurse & - & - \\
\hline diet & vegetarian & vegetarian & vegetarian & vegetarian & vegetarian & vegetarian \\
\hline
\end{tabular}

Conversations with these individuals indicated that personal passion and enthusiasm for plant-based cooking has been a primary motivator in starting the business, irrespective of cultural differences. Although none of the restauranteurs were vegans themselves, it was importantly noted that they either 
grew up as vegetarians, or were exposed to vegetarianism within their close social networks early in their lives. Being vegetarian themselves, or having been exposed to a vegetarian social network, the restauranteurs found it an easy and logical step to establish a restaurant business offering vegan and vegetarian dishes. In one instance, the family tradition of running a vegetarian restaurant for 40 years was an obvious motivator. However, for most, it was the observation of a limited number of vegetarian restaurants, and the lack of quality in existing restaurants, that inspired them to start the business. Overall, respondents were motivated to provide good quality food that also aligned with their own ethical stance - i.e. running a business that has a personal meaning - rather than for purely financial reasons.

All respondents perceived notable growth in the vegan market with an increase in the number of vegans, and demand for vegan restaurants as well as vegan options in existing restaurants. The drivers of this growth are seen to be the increasing consciousness of society's impact on the environment and animal welfare, but also the burgeoning health-conscious population who choose to adopt more vegan meals. This population also includes an older demography, who, under the guidance of their doctors, adopt a vegan diet. Uncertainty nevertheless remains as to whether this movement is a "fad" or one that is here to stay. Interestingly, none of the respondents were concerned with the establishment of a larger number of vegetarian/vegan restaurants. Rather, they perceive this as a positive development, which can help spread the idea of healthy eating. A couple of respondents added that competition, in other words, greater number of restaurants, only serve to help increase the quality of their own offering.

How the restauranteurs design vegan dishes is naturally governed by their perceptions of customer demand - motivations as well as demotivations to purchase vegan meals (see Table 2). Customers are seen to purchase vegan meals because they provide healthy options, which are fresh, natural (e.g. organic), and contain plenty of vegetable ingredients. This understanding compels the restauranteurs to be conscientious about where they source their ingredients from, often preferring small, local, and non-commercial suppliers that they can support. Nevertheless, this design strategy raises production costs, which can be undesirable among potential adopters, a paradox underscored by respondents who note that for some customers, eating vegan meals is a way to save money.

Table 2: Perceived motivations and demotivations of individuals to purchase vegan dishes.

\begin{tabular}{llll}
\hline Factors & Motivation & Demotivation & Design Considerations \\
\hline \hline healthy & $\mathrm{X}$ & & conscientious sourcing \\
high quality (fresh, organic etc.) & $\mathrm{X}$ & & conscientious sourcing \\
positive impact on environment & $\mathrm{X}$ & & conscientious sourcing \\
price & $\mathrm{X}$ & $\mathrm{X}$ & - \\
taste and texture of traditional dishes & & $\mathrm{X}$ & include faux meat (?) \\
doubts about nutritional benefits & & $\mathrm{X}$ & education program \\
doubts about satisfying hunger & & $\mathrm{X}$ & - \\
\hline
\end{tabular}

By and large, respondents aim to showcase the attractiveness or "sexiness" of vegan dishes, concurrently wanting to demonstrate that delicious food can indeed be produced without meat. Restauranteurs believe that customers may be discouraged if they compare the vegan dish to a traditional one, which might set certain expectations. Such expectations may relate to taste and texture, which, if deemed to be absent in the vegan meal, can demotivate its purchase. The inclusion of faux meat (i.e. imitation meat) is thus a popular practice in the industry. Nevertheless, the use of faux meat can concurrently demotivate some customers (particularly vegan customers) from opting for a vegan dish. This creates a second paradox that restauranteurs must deal with. 


\section{Strategies to Reach the Main Market}

All of the restauranteurs interviewed desired to have a larger customer base, and had subsequently developed strategies to meet this objective. These strategies were inevitably devised to reach out to the members of the main market, who are not vegan, with the ambition of overcoming their critical inhibitions to purchase vegan dishes. The first set of strategies centred on price incentives. In this manner, one respondent considered offering discounts on a given day of the week, although rejected this concept due to potential overcrowding. A further idea was to develop a collaboration scheme between vegetarian/vegan restaurants in the city to increase loyalty, and concurrently offering discounts to loyal customers.

A second set of strategies focused on alleviating passive rejection (i.e. rejection without having trialled the product) primarily based on the prejudice of the main market. One tactic employed to this end is the offering of tasting samples to passers-by to demonstrate the quality of vegan dishes. Two other tactics, in turn, rely on the design aspects of vegan dishes that aim to satisfy different sub-groups within the non-vegan main market. To appeal to more adventurous individuals, our respondents suggested the need to create dishes that increase the curiosity of non-vegans. This drive to innovate is coupled with a requirement for high quality to meet the demand of the more knowledgeable market that pursues new flavours and "honest food". By comparison, to attract more conservative members of the main market, the restauranteurs advocated the creation of dishes that emulate well known, traditional dishes. While the use of faux meat is often employed to meet this objective, one respondent mentioned the necessity to innovate vegan dishes that come close to the taste and texture of traditional meat dishes (e.g. vegetarian steak), in the absence of faux meat.

Creating greater awareness was a third overarching strategy employed by restauranteurs. One respondent noted her attempt to increase visibility through the distribution of flyers (a failed endeavour), and appearing on mainstream media (e.g. popular magazines and TV programs). Organizing events (e.g. concerts, wine tasting, and workshops on healthy eating) at the restaurant was another tactic to generate awareness. A few of the respondents also mentioned their reliance on word of mouth marketing, and the help of certain NGOs in this effort. The use of online channels (e.g. restaurant websites and Facebook pages) to garner greater interest surprisingly varied between the restaurants, ranging from regular and purposeful activity to none or very little. Interestingly, only one of the six restaurant owners underscored a detailed market segmentation strategy. This restaurant opened a new retail outlet (its fourth) in the food court of a shopping mall to attract a different demography, while simultaneously planning the launch of a pre-packaged meal product line that targets corporate consumer segments.

As for strategies designed to attain the repeat purchase of vegan dishes, the respondents suggested that the "vegan" characteristic of these dishes will attract their initial purchase by vegans (i.e. the early market), although it is another factor, namely, "health" that will guarantee repeated purchase. For nonvegans, by comparison, it could be proposed that it is "curiosity" that drives the initial purchase, but it is an additional factor, namely, "taste" that will ensure repeated purchase.

\section{Conclusions}

The findings of this research project will have implications for practitioners who endeavour to start a new business or expand an existing business through vegan food innovations. For these businesses, crossing the chasm is the biggest challenge in entering the main market with their new products. This is a critical consideration for many sustainable products that face segmented demand from the market. Moore (1991) suggests that to navigate successfully across this divide, firms should first, identify a small, niche segment in the main market; second, identify the "problem" experienced by this niche segment that a new product or service can resolve; and third, redesign the innovation to resolve the problem of the niche segment, thereby gaining an important foothold in the main market by attracting members of this initial segment to the innovation (e.g. Egmond et al. 2006). This process has been examined in some earlier studies, albeit within the high-tech product context. In their analysis of the 
diffusion of telehealth innovation into hospitals, Cho et al. (2008), for instance, illustrate that reaching the main market requires the reworking of the product and business strategy, originally devised to target the early market.

This study suggests that businesses entering the vegan food retail sector can benefit from viewing the main market as comprising two groups of individuals. Members of the first group are non-vegans who are more adventurous and seek new flavours and novel dishes. Vegan food innovations that are highly creative and also high in quality are likely to appeal to this group. Following Moore's suggestion, this group may indeed represent the niche segment within the main market that vegan food retailers should target at the outset. The second group constituting the main market are likely to be more conservative non-vegans, for whom highly creative vegan dishes may not offer an attractive value proposition. Rather, the findings our this study propose that designing vegan dishes that are more familiar to these individuals is a more effective tactic, for instance, with the inclusion of faux meat in dishes to provide taste and texture qualities sought by these individuals. It is additionally proposed that conservative members of the main market are more likely to adopt vegan food innovations when the more adventurous members adopt before them. In this manner, the latter offers an important point of reference that helps alleviate inherent prejudices. These findings overall suggest that the mainstream market can be divided into two sub-groups, which adopt innovations sequentially. This is an important extension of the existing conceptual understanding of the market within which innovations disseminate, as it allows firms to sequentially reformulate the value proposition of products/services for the early adopters, the curious sub-group of the main market, followed by the conservative subgroup.

Further extensions of this study can help verify these findings, while concurrently overcoming the limitations of the study bestowed by the number of cases and the unilateral perspective on the phenomenon in particular. To complement the responses acquired from business owners, conversations with both vegan (i.e. members of the early market) and non-vegan (i.e. members of the main market) customers can provide highly valuable insights. Specifically, the factors that motivate (and demotivate) the adoption of vegan food innovations, as acquired directly from these individuals, can help arrive at a more comprehensive understanding of strategic considerations.

\section{References}

Adams, C. J. (2010). Why feminist-vegan now? Feminism \& Psychology, 20(3): 302-317.

Bass, F. M. (1969). New product growth model consumer durables. Management Science, 15(5): 215227.

Berger, T. (2001). Agent-based spatial models applied to agriculture: A simulation tool for technology diffusion, resource use changes and policy analysis. Agricultural Economics, 25(2-3), 245-260.

Chandrasekaran, D., \& Tellis, G. J. (2011). Getting a grip on the saddle: Chasms or cycles? Journal of Marketing, 75(July): 21-34.

Cole, M., \& Morgan, K. (2011). Vegaphobia: derogatory discourses of veganism and the reproduction of speciesism in UK national newspapers. The British Journal of Sociology, 62(1): 134-153.

Deckers, J. (2009). Vegetarianism, sentimental or ethical? Journal of Agric Environ Ethics, 22: 573597.

Easingwood, C., Moxey, S., \& Capleton, H. (2006). Bringing high technology to market: Successful strategies employed in the worldwide software industry. Journal of Product Innovation Management, 23: 498-511.

Edmondson, A. C., \& McManus, S. E. (2007). Fit in methodological management. Academy of Management Review, 32(4):1155-1179.

Egmond, C., Jonkers, R., \& Kok, G. (2006). A strategy and protocol to increase diffusion of energy related innovations into the mainstream of housing associations. Energy Policy, 34: 4042-4049.

Eisenhardt, K. M. (1989). Building theories from case study research. Academy of Management Review, 14(4): 532-550.

Faiers, A., \& Neame, C. (2006). Consumer attitudes towards domestic solar power systems. Energy Policy, 34(14): 1797-1806. 
Goldenberg, J., Libai, B., \& Muller, E. (2002). Riding the saddle: How cross-market communications can create a major slump in sales. Journal of Marketing, 66(2): 1-16.

Golder, P. N., \& Tellis, G. J. (2004). Growing, growing, gone: Cascades, diffusion, and turning points in the product life cycle. Marketing Science, 23(2): 207-218.

Gombault, A., Allal-Chérif, O., \& Décamps, A. (2016). ICT adoption in heritage organizations: Crossing the chasm. Journal of Business Research, 69: 5135-5140.

Greenebaum, J. (2012). Veganism, identity and the quest for authenticity. Food, Culture \& Society, $15: 1,129-144$.

Guseo, R., \& Guidolin, M. (2011). Market potential dynamics in innovation diffusion: Modelling the synergy between two driving forces. Technological Forecasting \& Social Change, 78: 13-24.

Hienerth, C., \& Lettl, C. (2011). Exploring how peer communities enable lead user innovations to become standard equipment in the industry: Community pull effects. Journal of Product Innovation Management, 28(1):175-195.

Jee, S.-J., \& Sohn, S.-Y. (2015). Patent network based conjoint analysis for wearable device. Technological Forecasting \& Social Change, 101: 338-346.

Judge, M., \& Wilson, M. S. (2015). Vegetarian Utopias: Visions of dietary patterns in future societies and support for social change. Futures, 71: 57-69.

Kim, T., Hong, J.-S., \& Lee, H. (2016). Predicting when the mass market starts to develop: The dual market model with delayed entry. IMA Journal of Management Mathematics, 27: 381-396.

Kohli, R., Lehmann, D. R., \& Pae, J. (1999). Extent and impact of incubation time in new product diffusion. Journal of Product Innovation Management, 16: 134-144.

LeRouge, C., \& Garfield, M. J. (2013). Crossing the telemedicine chasm: Have the U.S. barriers to widespread adoption of telemedicine been significantly reduced? International Journal of Environmental Research and Public Health, 10: 6472-6484.

Libai, B., Mahajan, V., \& Muller, E. (2009). Can you see the chasm? In Review of Marketing Research, 38-57.

Mahajan, V., \& Muller, E. (1998). When is it worthwhile targeting the majority instead of the innovators in a new product launch? Journal of Marketing Research, 35(4): 488-495.

Miles, M. B., Huberman, A. M., \& Saldana, J. (2014). Qualitative Data Analysis (3rd ed.). SAGE Publications, Inc.: United States of America.

Moore, G. A. (1991). Crossing the Chasm. New York, Harper Business.

Muller, E., \& Yogev, G. (2006). When does the majority become a majority? Empirical analysis of the time at which main market adopters purchase the bulk of our sales. Technological Forecasting \& Social Change, 73: 1107-1120.

Peres, R., Muller, E., \& Mahajan, V. (2010). Innovation diffusion and new product growth models: A critical review and research directions. International Journal of Research in Marketing, 27: 91106.

Potts, A., \& Parry, J. (2010). Vegan sexuality: Challenging heteronormative masculinity through meat-free sex. Feminism \& Psychology, 20(1): 53-72.

Rogers, E. M. (1995). Diffusion of Innovations. (4th ed.). New York, NY, Free Press.

Ruby, M. B. (2012). Vegetarianism: A blossoming field of study. Appetite, 58: 141-150.

Sanderford, A. R., Overstreet, G. A., Beling, P. A., \& Rajaratnam, K. (2015). Energy-efficient homes and mortgage risk: Crossing the chasm at last? Environ Syst Decis, 35:157-168.

Sawng, Y.-W., Om, K., Shin, B., \& Lee, J. (2009). Discontinuous innovation and market chasm: The case of digital convergence services. Journal of Research and Practice in Information Technology, 41(3): 219-239.

Seitz, C. S., Beuttenmüller, O., \& Terzidis, O. (2015). Organizational adoption behavior of CO2saving power train technologies: An empirical study on the German heavy-duty vehicles market. Transportation Research Part A, 80: 247-262.

Singer, P. (1990). Animal Liberation. 2nd ed. The New York Review of Books, New York (USA).

Sroufe, R., Curkovic, S., Montabon, F., \& Melnyk, S. A. (2000). The new product design process and design for environment: "Crossing the chasm". International Journal of Operations \& Production Management, 20(2): 267-291.

Taylor, S. (2013). Vegans, freaks, and animals: Towards a new table fellowship. American Quarterly: 757-767. 
Taylor, J. R., Moore, E. G., \& Amonsen, E. J. (1994). Profiling technology diffusion categories. Journal of Business Research, 31: 155-162.

Vakratsas, D., \& Kolsarici, C. (2008). A dual-market diffusion model for a new prescription pharmaceutical. International Journal of Research in Marketing, 25: 282-293.

Wejnert, B. (2002). Integrating models of diffusion of innovations: A conceptual framework. Annual Review of Sociology, 28: 297-326.

Williams, J. (2008). Predicting an American future for cohousing. Futures, 40: 268-286.

Yin, B. R. K. (1994). Case Study Research: Design and Methods.

Zhang, D., \& Di Benedetto, C. A. (2010). Radical fashion and radical fashion innovation. Journal of Global Fashion Marketing, 1-4: 195-205. 